\title{
Electronic states on a fractal: Inverse-iteration method
}

\author{
P. Kappertz \\ Fachbereich Physik and Institut für Chemie und Biologie des Meeres, Universität Oldenburg, D-26111 Oldenburg, Germany \\ R. F. S. Andrade \\ Instituto de Fisica, Universidade Federal da Bahia, 40210-340 Salvador, Brazil \\ H. J. Schellnhuber \\ Potsdam-Institut für Klimafolgenforschung, D-14473 Potsdam, Germany \\ (Received 15 July 1993; revised manuscript received 24 January 1994)
}

\begin{abstract}
The quantum dynamics of electrons on the Koch fractal is investigated within the framework of a tight-binding approximation. The invariant sets of an exact real-space renormalization procedure play a crucial role for the mainly analytical methods employed. Inverse iteration of the critical fixed points is the key to the construction of dense spectral subsets. This approach is confirmed by numerical determination of periodic orbits of the decimation map. As a corollary, a classification scheme for the quantum states of the system is proposed. This scheme is directly related to the 4-adic representation of the rotation number of the wave function.
\end{abstract}

\section{INTRODUCTION}

Fractal geometry ${ }^{1,2}$ has provided us with many new well-defined structures, which can be either used to mimic real objects like trees, or to investigate the properties of familiar dynamics, like the one described by Schrödinger's equation, within a novel setting. The latter effort is certainly rewarding as it sheds new light on the intrinsic character of processes, which seem well understood when standard conditions like translational symmetry prevail.

Quantum-mechanical next-neighbor hopping on the Koch curve is a nice example for rich behavior induced by the fractality of the substrate: Various types of spectral scaling behavior are present; localized, critical, and extended wave functions alternate in a complicated way; an infinity of quantum levels condense to form a superlocalized phase under certain circumstances. The model was introduced some years ago $^{3}$ by mounting an adequate Hamiltonian on the fractal substrate proposed by Gefen et al. ${ }^{4}$ in a different context. A first analysis was carried out by means of a transfer matrix method, while the results were recently corroborated and extended using an exact renormalization group technique for the evaluation of the pertinent Green's functions. ${ }^{5}$

The purpose of this paper is to present a concise classification scheme for the quantum states of the system. These states are most conveniently labeled by their quantum rotation number $\kappa$, which is directly related to the integrated density of states. ${ }^{6,7}$ For further details regarding this specific point and a general description of the model considered, we refer to the above-mentioned article. $^{5}$

As the Koch curve is a nonbranching fractal, our model can be mapped onto a linear chain with a limit-periodic distribution of hopping strengths. Therefore, the system investigated here is sort of intermediate between $1 D$ almost periodic Schrödinger operators ${ }^{8}$ and finitely ramified fractals. ${ }^{9}$ As shown in Ref. 3, the recurrence relations obtained by the transfer matrix approach permit the analytical determination of the main invariant sets [the fixed points (lines) of the corresponding map] in the three-dimensional parameter space governing the system. The inspection of these sets directly leads to an explanation of many properties of spectrum and wave functions. Other invariant sets (like the preimages of fixed points and periodic cycles), which cannot be expressed in closed form, may be computed by numerical methods.

As a matter of fact, all relevant physical information is contained in the following two-dimensional map: ${ }^{3}$

$$
\begin{aligned}
\xi_{N+1}= & \frac{1}{2}\left(w+w^{-1}\right)\left[\left(\xi_{N}^{2}-2\right)^{2}-2\right] \\
& +\frac{1}{2}\left(w-w^{-1}\right) \eta_{N} \xi_{N}\left(\xi_{N}^{2}-2\right), \\
\eta_{N+1}= & \frac{1}{2}\left(w+w^{-1}\right) \eta_{N} \xi_{N}\left(\xi_{N}^{2}-2\right)+\frac{1}{2}\left(\omega-\omega^{-1}\right)\left(\xi_{N}^{2} \eta_{N}^{2}+2\right) .
\end{aligned}
$$

Here $\xi_{N}$ is the trace of the transfer matrix $A_{N}$ for the wave amplitude vector in the $N$ th-order periodic approximation of the limit-periodic system. $\eta_{N}=\left(A_{N}\right)_{11}$ $-\left(A_{N}\right)_{22}$, and $w=1+\lambda E$ is a combination of crucial parameters, namely cross-hopping strength $\lambda$ and energy $E$.

The initial conditions for the map (1) are

$$
\begin{aligned}
& \xi_{1}(E, \lambda)=-\left(\omega+\omega^{-1}\right)+\omega\left(E^{2}-2\right)^{2}, \\
& \eta_{1}(E, \lambda)=\left(\omega-\omega^{-1}\right)-\omega^{-1}\left(E^{2}-2\right)^{2},
\end{aligned}
$$

defining the "launching surface"

$$
\omega(\xi+\eta)+2=0
$$

in $\xi-\eta-\omega$ space.

For any $N$ th-order approximation the spectrum $\sigma_{N}$ is a finite collection of subbands. As proved in Ref. 3, each of these subbands splits into four new intervals if we go from a given generation to the next (see Fig. 1).

Therefore the number of subbands is $4^{N}$ in the $N$ thorder approximation, i.e., each subband embraces $1 / 4^{N}$ 


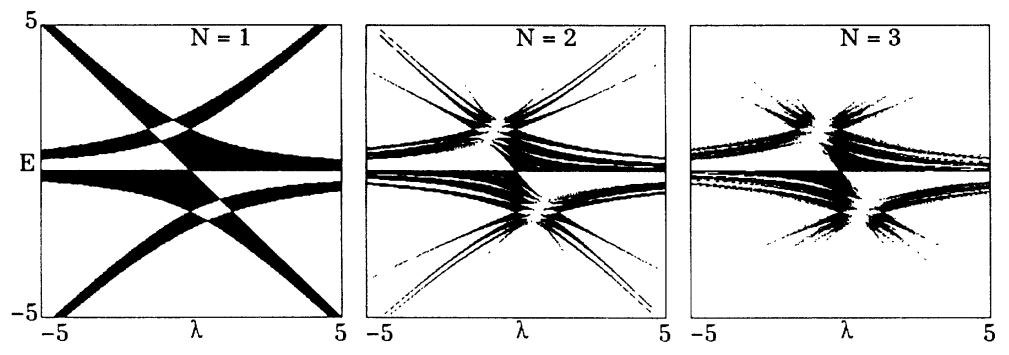

FIG. 1. Family of energy spectra as produced by variation of $\lambda$. Periodic approximation. of all the (extended) states associated with the spectrum $\sigma_{N}$. The perpetual splitting process produces a spectrum $\sigma(E)$ of Cantor-set type in the limit $N \rightarrow \infty$.

Neither the energy nor the ordinary wave number $k=(2 E m)^{1 / 2} / \hbar$ are good quantum numbers for the identification of definite electronic states through successive approximations. To keep track of a specific quantum level we use instead the quantum rotation number $\kappa \equiv \kappa(E)$, which is a strictly increasing function on the spectrum, defined as ${ }^{6,7}$

$$
\kappa(E) \sim \int_{-\infty}^{E} \rho(\bar{E}) d \bar{E} .
$$

Note that, in general, $E_{N+1}(\kappa) \neq E_{N}(\kappa)$, i.e., the energy associated with a particular value of $\kappa$ will slightly oscillate with the order of the approximation. Nevertheless, in the limit $N \rightarrow \infty$ the relationship between $\kappa$ and $E$ is well defined, and our classification scheme assigns to $\kappa$ the corresponding quantum state and energy eigenvalue in a clear-cut way.

\section{INVARIANT SETS AND THE SPECTRUM}

The invariant sets of the dynamical system (1) are directly related to several characteristic traits of spectrum and quantum states. The most relevant sets here are the fixed points, their preimages, and periodic cycles of higher order.

In the general case, i.e., $\omega \neq \omega^{-1}$, the critical fixed points are given by ${ }^{3}$

$$
\begin{aligned}
& \xi_{ \pm}^{*}=-\frac{1}{4}\left(\omega+\omega^{-1}\right) \pm \frac{1}{4}\left[\left(\omega+\omega^{-1}\right)^{2}+16\right]^{1 / 2} \\
& \eta^{*}=\frac{1}{2}\left(\omega-\omega^{-} 1\right)
\end{aligned}
$$

These points define two fixed lines in the enlarged $\xi-\eta-\omega$ space, which intersect the surface of initial conditions (3). The associated $\omega$-values directly yield two different parameter couples $\left(E^{*}, \lambda^{*}\right)$. The invariance of all these entities under the mapping (1) implies that $E^{*}$ is a spectral value for the cross-hopping strength $\lambda^{*}$ and the corresponding quantum state is critical (power-law distributed).

One of the results presented in this paper is the fact that an infinity of additional spectral values with critical wave functions can be generated from the fixed points $\left(\xi_{ \pm}^{*}, \eta^{*}\right)$ in a straightforward way: The highly nonlinear dynamical system (1) has the remarkable property of being invertible in closed form, i.e.,

$$
\begin{aligned}
& \xi_{N}= \pm\left[2 \pm\left[4-\frac{Z_{N+1}}{Y_{N+1}}\right]^{1 / 2}\right]^{1 / 2}, \\
& \eta_{N}=\frac{\xi_{N+1}+\frac{w+w^{-1}}{2}\left(\frac{Z_{N+1}}{Y_{N+1}}-2\right)}{\frac{w-w^{-1}}{2}\left[2 \pm\left[4-\frac{Z_{N+1}}{Y_{N+1}}\right]^{1 / 2}\right]^{1 / 2}\left[4-\frac{Z_{N+1}}{Y_{N+1}}\right]^{1 / 2}} .
\end{aligned}
$$

This formula defines four different preimages of a given point $\left(\xi_{N+1}, \eta_{N+1}\right)$ as a consequence of the twofold sign option on the right-hand side of the first line. Note that the second choice simultaneously determines the sign on the right-hand side of the second line. $Z_{N+1}$ and $Y_{N+1}$ are given by

$$
\begin{aligned}
& Z_{N+1}=4-\xi_{N+1}^{2}+2\left(\omega-\omega^{-1}\right)\left(\eta_{N+1}-\omega+\omega^{-1}\right), \\
& Y_{N+1}=2+\frac{\omega+\omega^{-1}}{2} \xi_{N+1}+\frac{\omega-\omega^{-1}}{2} \eta_{N+1} .
\end{aligned}
$$

Using Eqs. (6) and (7) we can determine the preimages with respect to (1) of any given point in parameter space. The backward iteration is particularly useful when applied to the fixed lines defined by $\left(\xi_{ \pm}^{*}, \eta^{*}\right)$. Due to the multiple choice of sign in Eq. (6), each of the two fixed lines has four different preimages which cross the manifold of initial conditions (3) at different locations. In this way we obtain a set of critical points that will be mapped onto a fixed point in $\xi-\eta-\omega$ space under one forward iteration.

By further backward iteration of the fixed lines and

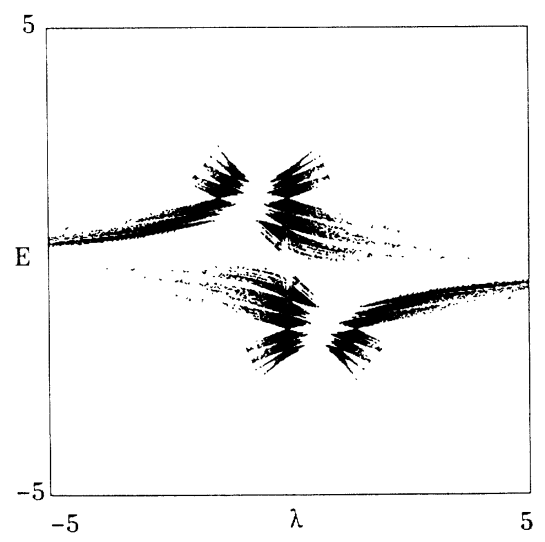

FIG. 2. Family of energy spectra. Subset derived from inverse iteration of the critical fixed points (lines). 


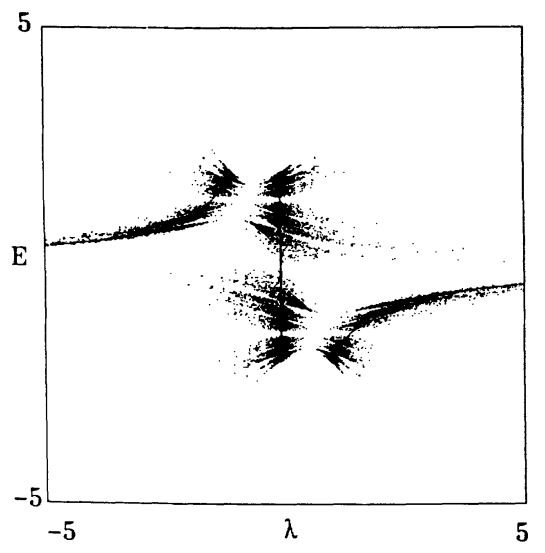

FIG. 3. Family of energy spectra. Subset calculated from periodic orbits of the renormalization map.

determination of the intersections with the launching surface, we can produce an exponentially growing number of spectral values $\hat{E}$ valid for the associated system parameter $\hat{\lambda}$. As the stability properties of the fixed lines with respect to (1) do not depend on $\omega$, all the corresponding quantum states will have the same critical character. Figure 2 displays the locations of the $(\hat{E}, \hat{\lambda})$-couples obtained by backward iteration up to the seventh order.

$P$-periodic cycles of the map (1) are defined by the equation

$$
\left(\xi_{N+P}, \eta_{N+P}\right)=\left(\xi_{N}, \eta_{N}\right) \text { for all } N \in \mathbf{N} .
$$

In this context, the fixed points appear as cycles of period 1. For finding such $P$-cycles and their intersections with the initial manifold in $\xi-\eta-\omega$ space there is no direct analytic procedure. We have determined these sets using a combination of computer algebra standard and rootfinding methods. Figure 3 shows the results, namely all the pairs $(\widetilde{E}, \tilde{\lambda})$ corresponding to cycles up to period 8 . The periodicity property guarantees that $\widetilde{E}$ is a spectral value for the cross-hopping strength $\tilde{\lambda}$.

Comparing Figs. 2 and 3 with Fig. 1 we find that both the spectral subset generated from backward iteration of the fixed points and the one produced by determining periodic orbits tend to be dense in the full spectrum. This means, in particular, that all the quantum levels of our fractal hopping Hamiltonian can be constructed, in principle, from exact backward iteration and closure. (The only possible exceptions, namely the singular spectral levels associated with superlocalized states, can be determined directly anyway.)

\section{THE CLASSIFICATION SCHEME}

As explained above, the quantum states of our model can be labeled in an unambiguous way by the quantum rotation number $\kappa(E)$, which represents the extension of the familiar Bloch wave vector to almost-periodic systems. $\kappa(E)$ is proportional to the integrated density of states. Therefore its $N$ th-order approximation $\kappa_{N}(E)$ can be easily determined by counting the number of full sub- bands to the right of the specific $E$-value considered and by adding an adequate remainder.

Due to the fourfold band splitting in the process of periodic approximations of our limit-periodic system, the 4-adic representation of $\kappa$ is the most natural one. The corresponding infinite string of figures from the set $\{0,1,2,3\}$ encodes precisely the location of the quantum level in question within the subband hierarchy of the spectrum.

Using this approach, we have analyzed the quantum rotation numbers of the states, which can be generated precisely from the critical fixed lines and their preimages. Let $N_{B} \in \mathbf{N}$ denote the backward iteration depth. Then we can summarize our findings as follows: (i) The 4-adic representation of $\kappa$ has a finite head, whose number of digits equals $N_{B}+1$; (ii) this head is followed by a periodic tail formed by infinite repetition of the string $\{03\}$ or the string $\{12\}$; (iii) $\kappa$ can be expressed as a rational number $p / q$, where $q=10 \times 4^{N_{B}}$. A similar analysis of the $\kappa$ values produced by periodic cycles leads to the following results. (a) The 4-adic representation of $\kappa$ has a periodic tail composed of strings of length $2 \times P$, where $P$ is the peroid of the cycle; (b) in the rational representation, all $\kappa$ values derived from cycles with the same period have a characteristic common denominator.

By construction, the states discussed so far have to be critical. This can be confirmed by the independent method of the band-gap ratio. ${ }^{10}$ On the basis of this fact and the spectral properties detected in the previous sections we conjecture that all the states with $\kappa$-values belonging to one of the classes described above are critical (exotic). Our numerical analysis of randomly chosen wave functions indicates that this hypothesis is correct.

\section{CONCLUSIONS}

Quantum hopping on the Koch fractal belongs to the small class of almost periodic problems, which can be successfully treated by analytical methods. In this paper we have presented additional results regarding the composition of the spectrum and the nature of the associated, at least algebraically bounded states. Our approach was mainly based on the following properties of the model: (1) There exists an exact real-space renormalization scheme for the quantum dynamics; (2) this scheme can be inverted for backward iteration in parameter space; (3) the invariant sets of the renormalization scheme are generic with respect to variation of the cross-hopping strength.

As a direct consequence, a dense subset of the spectrum can be constructed precisely from the renormalization fixed points. Our approach resembles the way Julia sets of rational complex maps may be generated by the inverse iteration method. ${ }^{11}$ The system investigated here thus belongs to the rather small class of aperiodic tightbinding models amenable to rigorous analysis. ${ }^{12}$ Regarding the methods employed, there is, in particular, much conceptual overlap with Schwalms' interesting work.

By combining backward iteration with subband bookkeeping we are also able to determine the exact rotation numbers for all the quantum states associated with the 
spectral subset. This leads to the proposition of a general classification scheme for the wave functions of the system.

We feel that the most pertinent features of the quan- tum Koch fractal are now fairly understood. What remains to be done, however, is the construction of vigorous mathematical proofs for a number of intricate details.
${ }^{1}$ B. B. Mandelbrot, The Fractal Geometry of Nature (Freeman, New York, 1983).

${ }^{2}$ K. Falconer, Fractal Geometry (Wiley, Chichester, 1990).

${ }^{3}$ R. F. S. Andrade and H. J. Schellnhuber, Europhys. Lett. 10, 73 (1989).

${ }^{4}$ Y. Gefen, B. B. Mandelbrot, and A. Aharony, Phys. Rev. Lett. 45, 855 (1980).

${ }^{5}$ R. F. S. Andrade and H. J. Schellnhuber, Phys. Rev. B 44, 13213 (1991).

${ }^{6}$ R. Johnson and J. Moser, Commun. Math. Phys. 84, 403 (1982).

${ }^{7}$ B. Simon, Adv. Appl. Math. 3, 463 (1982).

${ }^{8}$ J. B. Sokoloff, Phys. Rep. 126, 189 (1985); M. Kohmoto, L. P.
Kadanoff, and C. Tang, Phys. Rev. Lett. 50, 1870 (1983); S. Ostlund, R. Pandit, D. Rand, H. J. Schellnhuber, and E. D. Siggia, ibid. 50, 1873 (1983).

${ }^{9}$ R. Rammal, J. Phys. (Paris) 45, 191 (1984).

${ }^{10}$ H. J. Schellnhuber and H. Urbschat, Phys. Rev. Lett. 54, 588 (1985).

${ }^{11}$ H. O. Peitgen and P. H. Richter, The Beauty of Fractals (Springer, Berlin, 1986).

12J. Ashraff and R. B. Stinchcombe, Phys. Rev. B 37, 5723 (1988); R. B. Capaz, B. Koiller, and S. L. A. de Queiroz, ibid. 42, 6402 (1990); W. A. Schwalm and M. K. Schwalm, Physica A 185, 195 (1990); W. A. Schwalm and M. K. Schwalm, Phys. Rev. B 47, 7847 (1993). 